\title{
Uso de hidroxiapatita como recubrimiento en odontología. Una revisión breve.
}

\section{Use of hydroxyapatite coating in dentistry. A brief review.}

\author{
Rodrigo Rojas ${ }^{1 a}$ (iD) Natalia Sepúlveda ${ }^{1 a}$ (iD) Ricardo Cartes-Velásquez ${ }^{2 b}$ (iD)
}

\section{RESUMEN}

Desde principios del siglo XX la ingeniería de los tejidos ha desarrollado múltiples estudios relacionados como respuesta a la búsqueda de biomateriales similares al tejido óseo, intentando encontrar solución a los fracasos de implantación. Dentro de los biomateriales más importantes y con mayor sustento científico que existe, se encuentra la hidroxiapatita (HA). El objetivo de este artículo es revisar conceptos básicos y la evidencia en torno al uso de recubrimientos de HA en odontología. La aplicación de HA ha mostrado excelentes resultados, en ambientes tanto in vitro como in vivo, convirtiéndose en una excelente alternativa como coadyuvante en la cicatrización, mostrando óptimos resultados respecto a la calidad ósea, para terapias periodontales, elevaciones de seno o rehabilitaciones sobre implante.

El objetivo de este artículo es revisar conceptos básicos y evidencia en torno al uso de HA como recubrimiento en odontología.

\section{ABSTRACT}

Since the beginning of the 20th century, tissue engineering has developed multiple related studies in response to the search for biomaterials similar to bone tissue, trying to find a solution to implantation failures. Hydroxyapatite $(\mathrm{HA})$ is one of the most important and scientifically supported biomaterials that exists. The objective of this article is to review basic concepts and the evidence about the use of HA coating in dentistry. The application of HA has shown excellent results, in both in vitro and in vivo environments, becoming an excellent alternative as an aid in healing, showing optimal results regarding bone quality, for periodontal therapies, sinus elevations or implant restorations.

The aim of this article is to review basic concepts and evidence around the use of HA as a coating in dentistry.
1. Facultad de Odontología Universidad Andrés Bello, Concepción, Chile.

2 Fundación Kimntrum, Concepción, Chile.

a. Interno en Odontología

b. Doctor en Ciencias Médicas

Correspondencia:

Dr. Ricardo Cartes-Velásquez

Beltrán Mathieu 7, Concepción, Chile.

Correo electrónico:

cartesvelasquez@gmail.com

PALABRAS CLAVE:

Hidroxiapatita; injerto; recubrimiento; regeneración ósea; odontología

\section{KEYWORDS:}

Hydroxyapatite; graft; coating; bone regeneration; dentistry 


\section{INTRODUCCIÓN}

Entre todos los seres vivos, el humano es el único ser cuya tasa de regeneración es inversamente proporcional a su filogenia evolutiva, siendo el hueso el único tejido (a excepción del tejido embrionario) en constante renovación y que posee íntegra su capacidad regenerativa. ${ }^{1} \quad \mathrm{La}$ remodelación ósea es constante durante toda la vida, no obstante, hay distintas injurias que afectan su integridad, tales como la vejez, infecciones, traumas, alteraciones, genéticas, vasculares, entre otras; haciendo necesario el uso de técnicas que estimulen la correcta reposición del tejido.

En este sentido, los implantes ortopédicos se han convertido en una excelente alternativa mejorando la calidad de vida de millones de pacientes cada año, obteniendo tasas de éxito entre un $90-95 \%$ en cirugías de cadera durante los primeros 10 años y $80-85 \%$ en los controles a los 20 años. ${ }^{2}$ Este resultado, se genera gracias a la capacitación del personal clínico, los nuevos diseños de prótesis, protocolos de profilaxis y controles de esterilidad. Sin embargo, a pesar de los alentadores resultados clínicos obtenidos a la fecha, persiste un margen de fracaso que aún se genera, siendo la causa más frecuente de falla la insuficiencia ósea alrededor del implante visualizada inmediatamente después de la implantación, especialmente en pacientes osteoporóticos e infecciones. ${ }^{2}$

Desde principios del siglo XX la ingeniería de los tejidos ha desarrollado múltiples estudios relacionados como respuesta a la búsqueda de biomateriales similares al tejido óseo, intentando encontrar solución a los fracasos de implantación. El enfoque de estas investigaciones se basa fundamentalmente en que los materiales sean capaces de cumplir los requerimientos mecánicos y biológicos a los que el hueso está constantemente sometido. Esta perspectiva se debe ofrecer como una eficiente alternativa libre de riesgos para los autoinjertos y aloinjertos, mediante la combinación de biomateriales y células para promover la regeneración ósea, ${ }^{3}$ utilizando mecanismos que permitan la migración celular, vascularización y difusión de los nutrientes. Dentro de los biomateriales más importantes y con mayor sustento científico que existe, se encuentra la hidroxiapatita (HA).

El objetivo de este artículo es revisar conceptos básicos y evidencia en torno al uso de HA como recubrimiento en odontología.

\section{Tejido óseo.}

El hueso es el componente principal del esqueleto y tiene como función la protección de órganos vitales, además de posibilitar la acción mecánica de la musculatura. ${ }^{1}$ Está constituido fundamentalmente por un componente orgánico, integrado por fibras colágenas y un componente inorgánico, a base cristales de HA. ${ }^{4}$

La matriz orgánica o sustancia osteoide sirve principalmente como reservorio de calcio, fósforo, proteínas y otros iones, que participan en la regulación de la diferenciación celular, y en la integridad y función del tejido óseo. ${ }^{1}$ La matriz orgánica presenta una constitución principalmente de proteínas, de colágeno en un $90 \%$, de los cuales un $95 \%$ es tipo I y menos del $5 \%$ es tipo $\mathrm{V}$; mientras que el resto correspondiente a proteínas no colagenadas tiene un $10 \%$ de proteoglicanos y glicoproteínas en menor medida. ${ }^{8}$

$\mathrm{Su}$ fase mineral, está constituida principalmente por hidroxiapatita, que tiene como función factores de dureza y rigidez del tejido. ${ }^{5}$ Estos cristales de HA están formados por fosfato tricálcico y carbonato cálcico, además de otras sales minerales como magnesio y flúor ${ }^{6}$ que conforman aproximadamente el $70 \%$ del peso del hueso, junto con un $5 \%$ de agua y un $25 \%$ de materia orgánica. ${ }^{7}$ Para cumplir con estos requerimientos 
funcionales, de protección y acción mecánica, es necesario que el hueso se encuentre en constante homeostasis entre reabsorción y aposición ósea, la cual renueva un $5 \%$ del hueso cortical y un $20 \%$ del trabecular al año, resultando en una renovación de un $5-10 \%$ del hueso total al año. ${ }^{6}$ Este proceso de regeneración ósea ocurre fisiológicamente durante toda la vida, y patológicamente, después de un trauma. Para que la regeneración ocurra, se necesitan 3 componentes claves: una señal osteoinductiva, un sustrato, que actúa como andamio para la nueva formación ósea (osteoconducción) y finalmente, células del huésped capaces de diferenciarse en células óseas (osteogénesis). ${ }^{8}$

Es así, como posterior a un trauma, se produce una respuesta inflamatoria y un hematoma inicial, donde encontramos hematíes, plaquetas y fibrina. Una vez finalizada la cascada de coagulación y formado el coágulo, las células presentes liberan interleuquinas $y$ factores de crecimiento, originando la migración de linfocitos, macrófagos, precursores de osteoclastos y células mesenquimales pluripotenciales. Estas señales moleculares promueven la diferenciación hacia células especializadas; endoteliales, fibroblastos, condroblastos y osteoblastos, dando origen a un nuevo tejido fibrovascular, que reemplazará al coágulo inicial. ${ }^{1}$

El hueso aparecerá inicialmente, en forma de una red constituida por trabéculas (hueso esponjoso primario), sustituido posteriormente por el hueso esponjoso secundario, sufriendo modificaciones en forma y tamaño en función de procesos de reabsorción y aposición ósea. Posterior a los 30 días del depósito osteoide, comienza la mineralización que finalizará a los 130 días en el hueso cortical y a 90 días en el trabecular. ${ }^{1}$

\section{Generalidades de Hidroxiapatita.}

La HA se puede hallar como un mineral y un material biológico formado por fosfato de calcio cristalino, de fórmula ideal $\mathrm{Ca}_{5}\left(\mathrm{PO}_{4}\right)_{3}(\mathrm{OH})$. En su forma rudimentaria, podemos encontrarlo como mineral formando parte de rocas sedimentarias y metamórficas, mientras que en su formato de biocristal se localiza acompañado de estructuras orgánicas como el colágeno. ${ }^{9}$

La HA pertenece a la familia de los ortofosfatos, y en su forma sintética, se considera el material utilizado por excelencia en cuanto a sustitutos óseos, ya que ambos han mostrado similitudes estructurales y funcionales, a diferencia de otros fosfatos de calcio, ${ }^{10}$ por lo que independiente de si su origen es natural o sintético hace referencia a la hidroxiapatita sintética como una de las opciones más utilizadas. ${ }^{11}$

Durante sus años de aplicación clínica se han encontrado excelentes resultados en cuanto a las propiedades descritas, en ambientes tanto in vitro como in vivo ${ }^{12}$ convirtiéndose en una excelente alternativa como coadyuvante en la cicatrización, mostrando óptimos resultados respecto a la calidad ósea, para terapias periodontales, elevaciones de seno o rehabilitaciones sobre implante. ${ }^{1}$

A pesar de la evidencia a favor en lo que respecta a su uso, desde la perspectiva de la biocompatibilidad aún faltan estudios que señalen de manera específica, los mecanismos de acción de la HA sobre la cicatrización tisular y el tipo, cantidad y calidad ósea obtenida para poder comparar con otras terapias regenerativas y permitir así al tratante tomar una decisión más certera en cuanto a los beneficios de las distintas terapias disponibles. ${ }^{1}$

Aun así, sigue asegurando una excelente osteoconductividad, que combinada con matrices poliméricas, puede mejorar las propiedades mecánicas del $\operatorname{sistema}^{13}$ y otorgar a la bioingeniería, la oportunidad de continuar el estudio de HA junto a otro 
materiales bioactivos para modificar las falencias en cuanto al uso como recubrimiento de elementos implantables.

Teniendo en cuenta las características proactivas de la HA sintética convencional, aún hay desafíos por cumplir en cuanto a los requisitos que la medicina regenerativa actual requiere. Sus problemas radican en las bajas propiedades mecánicas, débil biodegradabilidad e insatisfactoria osteoinductividad, ${ }^{9}$ por lo tanto, su uso se limitaría a implantes que no necesiten permanecer largo tiempo y soportar alta carga. $^{14}$

Inicialmente, debido a la poca disponibilidad existente en los bancos de hueso y los riesgos asociados a la transmisión de infecciones, el uso de HA estuvo enfocado en evitar traumas quirúrgicos al momento de extraer aloinjertos, utilizándose en defectos óseos creados por quistes y aumentos de rebordes alveolares. ${ }^{15}$ Los resultados ante estos defectos arrojaron resultados favorables, pues se comprobó una osteointegración en el $100 \%$ de los casos; y no solo eso, sino que el material fue encontrado altamente compatible con el tejido óseo, resultando en que las pequeñas alteraciones encontradas son inherentes al trauma quirúrgico y no como respuesta adversa del material. ${ }^{16}$

La HA tras ser implantada, mantiene su funcionalidad, al contrario del aloinjerto, el cual a pesar de su excelente biocompatibilidad, pierde entre un $50 \%$ a $100 \%$ del peso original del producto en los primeros 5 años. ${ }^{16}$ Es por ello que desde 1980 se ha utilizado para mejorar la osteointegración de implantes. ${ }^{17}$ y otros injertos, especialmente en cirugía ósea o dentales en donde se requiera como recubrimiento. ${ }^{11}$

\section{Uso de HA como recubrimiento en Odontología.}

En Odontología el uso de HA es amplio en lo que respecta a cicatrización ósea, ya que sus distintos formatos permiten reparar segmentos óseos específicos. Se puede incluso inmovilizar los fragmentos, dando al cirujano la posibilidad de modificar su forma, cuidando de no dañar las propiedades mecánicas del biomaterial con instrumentos de corte diamantados, convirtiéndose en un gran aporte dentro de especialidades tales como cirugía maxilofacial, periodoncia. ${ }^{18}$

En implantología dental el uso de dispositivos de titanio, ha sido durante un largo tiempo una alternativa terapéutica ampliamente aceptada entre los dentistas y pacientes como un método de reemplazo a las piezas dentarias perdidas. Esto se refleja en un anclaje firme al hueso que sirve para base de pónticos y prótesis teniendo ventajas en comparación a prótesis removibles, tales como durabilidad, comodidad y conveniencia para el paciente. No obstante, aún tienen defectos relacionados con su osteointegración total y el hecho de que sus propiedades mecánicas no coinciden con las del hueso, ${ }^{19}$ llegando a un porcentaje aproximado del $7 \%$ de fallas post implantación, debido principalmente a sobrecarga mecánica del hueso circundante al implante, una falla en el proceso de cicatrización o una infección del sitio operatorio. $^{20}$

Por lo anterior es que los investigadores buscan disminuir este porcentaje de fallas en el tratamiento, orientando sus estudios en utilizar la HA como sustrato para el recubrimiento de implantes. Cabe recalcar que ante procedimientos quirúrgicos en los cuales exista pérdida de tejido óseo, es importante evaluar de manera rigurosa la osteoinducción y la osteointegración de los materiales, debido a que si estos no cumplen con las características mencionadas, en vez de mejorar las condiciones del paciente, podrían causar efectos no deseados, tales como procesos de fibrosis que retardan o impiden la adecuada cicatrización osea hasta en un $30 \% .^{21}$

Tradicionalmente, los recubrimientos a base de HA tienen como objetivo mejorar la adhesión de dispositivos implantables 
sólidos gracias a sus propiedades antibióticas y osteoinductoras, que aceleran el proceso de cicatrización ósea en momentos tempranos a su implantación. ${ }^{2} \mathrm{Se}$ ha demostrado que este uso como recubrimiento con nanopartículas de HA y fosfatos de calcio (CaP) otorgan propiedades osteoconductoras, relacionado con señales primarias que permiten la adhesión celular y la posterior inducción y neogénesis tisular. ${ }^{19}$

Al ser recubiertos con $\mathrm{HA}$, los implantes adquieren una mayor resistencia a la corrosión y una menor liberación de iones metálicos, mientras que el crecimiento óseo se ve incrementado. Como resultado, se logra una reducción significativa en el tiempo de curación del paciente con menos pérdida de resistencia mecánica de los implantes. $^{12}$

El uso de nano-HA sintética debería mejorar la calidad y cantidad del hueso alveolar, ya que presenta buenas propiedades como biomaterial, tanto en biocompatibilidad, bioactividad, osteoconductividad y unión directa al hueso; lo que hace suponer que además de su uso como material de relleno, tiene propiedades favorables para su uso como recubrimiento. Al comparar el tamaño de las partículas utilizadas en el recubrimiento de HA, se observó que la nano HA (nHA) mejora la adhesión de los osteoblastos a la superficie del implante, promueve su proliferación y el posterior depósito de calcio para producir hueso nuevo, en comparación con la HA tradicional a escala de micra $(\mathrm{mHA}){ }^{20}$ Esta lógica, quedó demostrada en un estudio realizado en donde se buscaba combinar las propiedades de la plata (antimicrobianas) y la HA (mejora en la osteointegración), usadas en conjunto como recubrimiento sobre implantes de titanio. Los investigadores observaron que la adición de nHA o mHA aumentó la rugosidad de la superficie entre valores desde los $0.2 \mu \mathrm{m}$ a $2 \mu \mathrm{m}$, resultando en la fijación de osteoblastos sanos, los cuales fueron analizados posterior a 7 días al microscopio con aumento de 200x y a escala de $100 \mu \mathrm{m} .{ }^{20}$ En ese mismo sentido, un implante recubierto con HA tiene un área de superficie $70 \%$ mayor en comparación con el implante no cubierto, por ende, una superficie más grande de contacto en relación al tejido óseo que recibe este implante. ${ }^{23}$

Por otra parte, se ha demostrado que el recubrimiento de nanocristales de $\mathrm{HA}$ incorporados a otras partículas como el silicio (Si-nHA), promovió significativamente la regeneración ósea y osteointegración hueso-implante en comparación con la HA sintética original, esto se tradujo en una mejora en la diferenciación osteogénica y angiogénica, además de una alteración de la relación OPG / RANKL de células estromales de la medula ósea (BMSC) en diabetes mellitus (DM). Por otro lado, el revestimiento de $\mathrm{Si}$ nHA mejoró aún más la proliferación celular, la diferenciación osteogénica y angiogénica, además de reprimir la osteoclastogénesis de BMSC en DM. ${ }^{22}$

También se ha evaluado el recubrimiento de HA sobre implantes de Polieteretercetona (PEEK), demostrando que los implantes recubiertos con HA, exhibieron mejoras importantes en la regeneración ósea y la fijación del implante, en comparación con PEEK sin recubrimiento, ya que se observa un aumento significativo de la osteointegración, además de presentar ventajas específicas, capaces de mejorar los resultados quirúrgicos. Los análisis de la microtomografía de rayos $\mathrm{X}$ permitieron estudios tridimensionales del volumen óseo regenerado, lo que resultó en una representación visual completa de la respuesta de curación periimplantaria, la cual, para este caso significó un aumento de 2 veces en la regeneración ósea, lo que fue asociado al grado de cristalinidad de la HA, lograda por tratamientos térmicos. Esto permitió observar que los implantes recubiertos mostraran un mayor contacto entre el hueso y el implante que el grupo de PEEK sin recubrimiento luego de 18 semanas. ${ }^{10}$ 
Actualmente, se está trabajando sobre recubrimientos biomiméticos, más próximos a la estructura apatítica fisiológica $\mathrm{y}$, por tanto, con mejores resultados a nivel biológico. $^{24} \mathrm{La}$ consecuencia de esto se refleja en un efecto positivo en cuanto a la regeneración del tejido, lo que reduce el riesgo de infección bacteriana, disminuyendo así, la posibilidad de rechazo por parte del cuerpo, proporcionando finalmente una mejor estabilidad. ${ }^{23} \mathrm{Un}$ ejemplo de ello, es utilizar sustratos como Ti13Nb13Zr y Ti13Nb11Zr, los cuales obtienen capas de 1-2 $\mu \mathrm{m}$ de HAp nanoestructurada tras $3 \mathrm{~h}$ de inmersión en una solución supersaturada de iones calcio y fósforo. Finalmente, se obtienen capas de nanopartículas de HA de 50 y $200 \mathrm{~nm}$ de espesor, ${ }^{24}$ dando como consecuencia que este recubrimiento biomimetico se refleje en un efecto positivo en cuanto a la regeneración del tejido, reduciendo así el riesgo de infección bacteriana y la posibilidad de rechazo por parte del cuerpo, proporcionando una mejor estabilidad. ${ }^{23}$

Una revisión reciente muestra que HA y fosfato cálcico $(\mathrm{Ca}-\mathrm{Ph})$ son las superficies bioactivas más utilizadas en investigaciones que buscan mejorar la oseointegración de implantes de titanio y que ciertas superficies bioactivas tienen un efecto positivo sobre la osteointegración, aunque ciertas biomoléculas de recubrimiento parecen influir en la formación temprana de hueso periimplantario. A pesar de resultados prometedores, aún faltan más investigaciones para dilucidar los mecanismos que permitirán reducir el tiempo necesario para la osteointegración de los implantes dentales. ${ }^{25}$ Sin embargo, esta situación parece no haber cambiado desde otra revisión sistemática publicada en 2017 y que concluye que a pesar que los estudios experimentales han demostrado que los recubrimientos osteogénicos son eficaces para mejorar el contacto huesoimplante, siguen faltando estudios clínicos que prueben un real impacto en la atención de nuestros pacientes. ${ }^{26}$

\section{CONCLUSIONES}

El uso de HA como material de recubrimiento en odontología ha mostrado resultados positivos, tanto in vivo como in vitro, en sus aspectos mecánicos y especialmente biológicos. La aparición de nHA y superficies biomiméticas han mostrado mejoras respecto a formas previas de HA. Sin embargo, es necesario más y mejor evidencia clínica del impacto que tiene el uso de HA como recubrimiento en el tratamiento de los pacientes.

\section{Conflictos de Interés}

Sin Conflictos de Interés

\section{Financiamiento}

Ninguno

\section{REFERENCIAS}

[1] Suárez D. Basic principles in guided bone regeneration. Rev. ULA. 2012; 14(2):129.

[2] Arcos D, Vallet-Regí M. Substituted hydroxyapatite coatings of bone implants. J Mater Chem B. 2020; 8(1):1781-800.

[3] Mangano C, Scarano A, Perrotti V, Lezzi G, Piattelli A. Maxillary sinus augmentation with a porous synthetic hydroxyapatite and bovine-derived hydroxyapatite: a comparative clinical and histologic study. Int J Oral Max Impl. 2007; 22(6):980-6.

[4] Hernández R, Palma R, Piña $M$. Hidroxiapatita y sus aplicaciones. Rev Mex Fis. 1999;45(1) 144-7.

[5] García M, Reyes J. La hidroxiapatita, su importancia en los tejidos mineralizados y su aplicación biomédica. Tip Rev Espec Cienc Quím Biol. 2016; 9(2):90-5.

[6] Da Silva AS, dos Santos PL, Marquesi A, Queiroz TP, Margonar R, de Souza AP.Some aspects of bone remodeling around dental implants. Revista Clin Periodon Implantol Rehab Oral. 2018;11(1):49-53.

[7] Ramesh N, Moratti S, Diaz G. Hydroxyapatite-polymer biocomposites for bone regeneration: A review of current trends. Soc Biomat. 2017;106(5):2046-57.

[8] Fernández I, Gracia M, Pingarrón $M$, Jerez L. Physiological bases of bone 
regeneration in Histology and physiology of bone tissue. Med Oral Patol Oral Cir Bucal. 2006;11(1):47-51.

[9] Lauritano D, Limongelli L, Moreo G, Favia G, Carinci F. Nanomaterials for Periodontal Tissue Engineering: Chitosan-Based Scaffolds. A Systematic Review. Nanomaterials. 2020; 10(1):1-16.

[10] Durham J, Montelongo S, Ong J, Guda T, Allen M, Rabiei A. Hydroxyapatite coating on PEEK implants: Biomechanical and histological study in a rabbit model. Mater Sci Eng C Mater Biol Appl. 2016;68:9.

[11] Britel O. Modélisation et optimisation par la méthodologie des plan d'expériences de la sythense de l'hydroxyapatite phosphocalcique, du phosphate tricalcique apatitique, du phosphate de calicium apatitique carbonate 2006. Rev Université Mohammed. 2006; 2326(1):1168.

[12] Dehghanghadikolaei A, Fotovvati B. Coating Techniques for Functional Enhancement of Metal Implants for Bone Replacement: A Review. Materials 2019;12: 1795.

[13] Xiangfeng L, Minjun L. Design of hydroxyapatite bioceramics with micro/nano-topographies to regulate the osteogenic activities of bone morphogenetic protein-2 and bone marrow stromal cells. R Soc Chem. 2020;12(5):7284-300

[14] Sequeda L, Díaz J. Obtención de hidroxiapatita sintética por tres métodos diferentes y su caracterización para ser utilizada como sustituto óseo. Rev Colomb Cienc Quím Farm. 2012;41(1):50-66.

[15] Pereira G,Oliva M. Eficacia de la hidroxiapatita en la cicatrización de injertos óseos e implantes dentales: Una revisión sistemática de la literatura. Int JOdontostomat. 2016;10(3):373-80.

[16] Quintana J. Utilización de la hidroxiapatita en cirugía maxilofacial, Actualización bibliográfica. Rev Cubana Estomatol. 1998;35(1):1-6.

[17] Gallo J, Langova K, Havranek V, Chechova I. Poor survival of ABG I hipprosthesis in younger patients. Biomed Pap Med Fac Univ Palacky Olomouc Czech Repub 2008; 152(1):163-8.
[18] González R. Hidroxiapatita Porosa Coralina Hap-200 15 Años de Aplicaciones Clínicas. Rev CNIC. 2005;36(1):1-8.

[19] Ogle O, Byles N. Nanotechnology in Dentistry Today. West Indian Med J. 2020;63(4):344-8

[20] Nadhim R, Besinis A, Huirong L, Tredwin C, D.Handy. R. The biocompatibility of silver and nanohydroxyapatite coatings on titanium dental implants with human primary osteoblast cells. Mater Sci Eng $\mathrm{R}$ Rep. 2020;107:1-11.

[21] Jaramillo C, Rivera J, Echavarría A. Comparación de las propiedades de osteoconducción y osteointegración de una hidroxiapatita reabsorbible comercial con una hidroxiapatita reabsorbible sintetizada. Rev Colomb Cienc Pecu. 2009;22(2):117-30

[22] Lui L, XiuhuiThe W. Synergistic promotion of osseointegration by nanostructure design and silicon substitution of hydroxyapatite coatings in a diabetic model. J Mater Chem B. 2020;20(1):5-18.

[23] Pajor K, Pajchel L, Kolmas J. Hydroxyapatite and Fluorapatite in Conservative Dentistry and Oral Implantology. Materials (Basel). 2019; 12(17): 2683.

[24] Melero H, Fernández J, Guilemany J. Recubrimientos bioactivos: Hidroxiapatita y titania. Biomecánica 2011;19(1):35-8.

[25] López-Valverde N, Flores-Fraile J, Ramírez JM, Sousa BM, HerreroHernández S, López-Valverde A. Bioactive Surfaces vs. Conventional Surfaces in Titanium Dental Implants: A Comparative Systematic Review. J Clin Med. 2020;9(7):2047.

[26] Ghanem A, Kellesarian SV, Abduljabbar T, Al-Hamoudi N, Vohra F, Javed F. Role of Osteogenic Coatings on Implant Surfaces in Promoting Bone-To-Implant Contact in Experimental Osteoporosis: A Systematic Review and Meta-Analysis. Implant Dent. 2017;26(5):770-7.

\section{CÓMO CITAR ESTE ARTİCULO}

Rojas R, Sepúlveda N, Cartes-Velásquez R. Uso de hidroxiapatita como recubrimiento en odontología. Una revisión breve. Appli Sci Dent. 2020;1(3):7-13 DOI: 10.22370/asd.2020.1.3.2482 